\title{
Positron emission tomography defines metastatic disease but not locoregional disease in patients with malignant pleural mesothelioma
}

Raja M. Flores, MD ${ }^{a}$

Timothy Akhurst, $\mathrm{MD}^{\mathrm{b}}$

Mithat Gonen, $\mathrm{PhD}^{\mathrm{c}}$

Steven M. Larson, MD ${ }^{\mathrm{b}}$

Valerie W. Rusch, MDa
From the Thoracic Service, Department of Surgery, ${ }^{a}$ Nuclear Medicine Service, Department of Radiology, b and Biostatistics Service, Department of Epidemiology and Biostatistics, ${ }^{c}$ Memorial Sloan-Kettering Cancer Center, New York, NY.

Read at the Eighty-second Annual Meeting of The American Association for Thoracic Surgery, Washington, DC, May 5-8, 2002.

Received for publication May 21, 2002; revisions requested July 17, 2002; revisions received Nov 25, 2002; accepted for publication Dec 19, 2002.

Address for reprints: Raja M. Flores, MD, Thoracic Service, Department of Surgery, Memorial Sloan-Kettering Cancer Center, 1275 York Avenue, New York, NY 10021 (E-mail: floresr@mskcc.org).

J Thorac Cardiovasc Surg 2003;126:11-6

Copyright (C) 2003 by The American Association for Thoracic Surgery

0022-5223/2003\$30.00+0

doi:10.1016/S0022-5223(03)00207-1
Background: Computed tomography and magnetic resonance imaging often fail to predict resectability in patients with malignant pleural mesothelioma. Small studies suggest that fluorodeoxyglucose-positron emission tomography may improve staging. We analyzed our experience to determine more definitively the potential utility of fluorodeoxyglucose-positron emission tomography.

Methods: Patients with malignant pleural mesothelioma who underwent fluorodeoxyglucose-positron emission tomography scanning were identified from an institutional database. All patients fasted and received a minimum of $10 \mathrm{mCi}$ of F-18-fluorodeoxyglucose. Whole-body emission studies were acquired, followed by whole-body transmission studies, allowing iterative reconstruction. Blinded review of positron emission tomography scans was performed for clinical staging, which was then correlated with surgical and pathologic findings. Sensitivity and specificity were determined for tumor and nodal status.

Results: From 1998 to 2002, 63 patients underwent positron emission tomography scans, 60 preoperatively and 3 to assess disease recurrence after surgery. Increased fluorodeoxyglucose uptake was seen in all but 1 tumor, which was very early stage (IA). Positron emission tomography findings yielded sensitivities of only $19 \%$ and $11 \%$ for tumor and nodal status, respectively. However, a high standard uptake value in the primary tumor correlated with the presence of N2 disease. Positron emission tomography correctly identified supraclavicular N3 or M1 disease in 6 patients.

Conclusions: Positron emission tomography does not identify the local extent of tumor or mediastinal nodal metastases reliably but detects extrathoracic metastases, thereby obviating inappropriate thoracotomy. Further studies of the association between tumor standard uptake value and the presence of N2 disease are warranted.

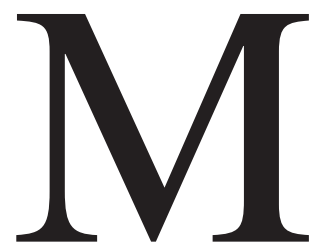

alignant pleural mesothelioma (MPM) is an uncommon tumor that is difficult to evaluate radiologically because of its propensity to infiltrate locally along tissue planes. Computed tomography (CT) and magnetic resonance imaging (MRI) are used to stage MPM but often fail to detect tumor invasion of the chest wall and diaphragm, as well as the presence of mediastinal nodal metastases. ${ }^{1,2}$ The treatment of early stage disease often involves 
surgical resection by either extrapleural pneumonectomy or pleurectomy/decortication. ${ }^{3,4}$ However, approximately $20 \%$ to $30 \%$ of patients undergo exploratory thoracotomy without resection because of the unreliability of current imaging modalities in identifying locally advanced or metastatic disease. ${ }^{2,5}$ Improved methods of determining resectability are needed to minimize the number of patients subjected to exploratory thoracotomy without resection.

Two studies suggested that MPM shows an increased uptake of fluorodeoxyglucose-positron emission tomography (FDG-PET) scanning, and that PET may be useful in staging this disease. ${ }^{6,7}$ However, these studies were too small to yield definitive information. We hypothesized that the functional tumor imaging provided by PET scanning might improve locoregional staging in patients undergoing preoperative evaluation or identify otherwise unsuspected extrathoracic disease, thereby avoiding inappropriate surgical exploration. Therefore, we reviewed our experience with FDG-PET scanning in MPM.

\section{Methods}

\section{Clinical Data}

All patients with biopsy-proven MPM who underwent FDG-PET scanning were identified from our institutional database. Clinical data on these patients were also extracted from a prospective institutional database. The pathologic diagnosis of mesothelioma was confirmed by review of histology and relevant immunohistochemical stains. All patients were considered for surgery if they had potentially resectable lesions identified by CT scan of the chest and upper abdomen and adequate cardiopulmonary function to tolerate the planned resection.

\section{PET Evaluation}

PET scans were acquired on dedicated bismuth germinate-based systems, including the GE Advance (GE Medicals Systems, Milwaukee, Wis), Siemens HR+, and Siemens Biograph scanners (CPS Innovations, Knoxville, Tenn). All patients were instructed to fast for 6 hours before the administration of FDG. After a minimum of 45 minutes postinjection of at least $10 \mathrm{mCi}$ of FDG, whole-body emission scans were performed, with rod sourcebased transmission scans to allow for iterative reconstruction with segmented attenuation correction.

A central review of all PET scans was performed by a single nuclear medicine physician who was blinded to all clinical information. Patients were clinically staged by PET using the International Mesothelioma Interest Group TNM staging system, the staging system for MPM which is now accepted by the American Joint Commission on Cancer and the Union Internationale Contre le Cancer. ${ }^{8-10}$ The standard uptake value (SUV) was calculated according to standard methods based on the uptake of FDG in grams per milliliter corrected for the injected dose of FDG adjusted for the patient's weight. To calculate the maximal SUV in the tumor, we thresholded the images electronically such that only the hottest voxel of the tumor was seen. A region of interest was drawn around the hottest voxel on the transaxial slice of the iteratively reconstructed images, and the SUV maximal value, corrected for body weight, was recorded.

\section{Statistical Methods}

The SUV was calculated for each tumor. The Wilcoxon test was then used to determine the relationship between tumor histologic subtype, and the mean SUV for all tumors was studied. A $P$ value of less than .05 was considered statistically significant.

Sensitivity and specificity on the basis of PET readings were calculated for tumor (T) and nodal (N) status. Sensitivity was defined as the number of patients with positive PET scan results divided by the total number of patients found to have positive results on surgical and pathologic examination. Specificity was defined as the number of patients with negative PET scan results divided by the total number of patients found to have negative results on surgical and pathologic examination. Positive predictive value was defined as the number of true positive findings by PET scan divided by the total number of positive PET scans. Negative predictive value was defined as the number of true negative PET findings divided by the total number of negative PET scan results.

Receiver operator characteristic (ROC) curves were calculated on the basis of SUV for both $\mathrm{N}$ and $\mathrm{T}$ status. The area under the curve (AUC) was calculated to determine the utility of SUV in predicting each status.

\section{Results}

\section{Demographics}

From April 1998 to February 2002, 63 patients with biopsyproven MPM underwent FDG-PET scanning. These patients included 52 men and 11 women, with a median age of 66 years (range $35-82$ years). There were 34 right-sided tumors and 29 left-sided tumors. There were 44 patients with tumors of epithelial histology, 16 with the mixed subtype and 3 with tumors of the fibrosarcomatous subtype.

\section{Types of Surgical Intervention}

Sixty patients underwent PET scans before definitive surgical intervention. Three patients underwent PET scans during follow-up to determine disease status after extrapleural pneumonectomy $(\mathrm{n}=2)$ or pleurectomy/decortication ( $\mathrm{n}$ $=1$ ). Twenty-six patients underwent extrapleural pneumonectomy, 10 patients underwent pleurectomy/decortication, 19 patients underwent exploratory thoracotomy without resection, 1 patient underwent wedge resection after pleurectomy, 2 patients underwent mediastinoscopy, and 2 patients underwent supraclavicular lymph node biopsy. At least 1 month before the PET scan, 10 patients had received induction chemotherapy and 18 patients had prior talc pleurodesis.

The patients who did not have a resection performed had disease invading the mediastinum or chest wall (T4 tumor). This reflects our institutional policy of offering patients exploration whenever preoperative imaging studies indicate potentially resectable disease and not performing an extrapleural pneumonectomy in cases unless all gross tumor 
TABLE 1. PET assessment of $T$ status compared with surgical and pathologic staging of $T$ status

\begin{tabular}{lcccc}
\hline & \multicolumn{3}{c}{ T status by PET } \\
\cline { 3 - 5 } & & T0-T3 & T4 & $\begin{array}{c}\text { Total no. } \\
\text { patients }\end{array}$ \\
\hline Surgical/pathologic T status & T0-T3 & 29 & 3 & 32 \\
Total no. patients & T4 & 17 & 4 & 21 \\
& & 46 & 7 & 53 \\
\hline
\end{tabular}

$P E T$, Positron emission tomography; $T$, tumor.

can be removed. We have a low threshold for exploration in this patient population because of the inaccuracies of CT scan and MRI in predicting resectability. We consider a patient as unresectable when there is diffuse chest wall invasion or disease invading mediastinal structures (eg, the vena cava) that would not be removed by an extrapleural dissection. Our goal in surgery is to rid the patient of all gross disease. If this is not possible with an extrapleural pneumonectomy, then the procedure is not performed, regardless of the feasibility.

\section{Analysis of PET Scan Results}

Of the patients who underwent PET scans before surgical intervention, 59 of 60 had FDG-18 uptake in the primary tumor. The median SUV of the primary tumor was 6.6 (range 1.7-23). The single patient who had no increased uptake on PET scan was found to have minimal parietal pleural disease (stage IA) at thoracoscopy.

PET findings were correlated with tumor histology. The mean SUV for epithelial tumors $(\mathrm{n}=39)$ was $6.67( \pm 2.78)$ and $7.37( \pm 5.82)$ for the mixed histology tumors $(\mathrm{n}=14)$. These were not significantly different $(P=.88)$. There were only 2 sarcomatous tumors with SUVs of 3.4 and 4.1 .

Of the 53 patients who underwent both FDG PET evaluation of T status and surgical and pathologic evaluation, 21 ultimately had unresectable tumors as the result of tumor invading the chest wall or mediastinum (T4 status), as shown in Table 1. The sensitivity of PET in identifying T4 status was 19\% (95\% confidence interval [CI]: 5\%-42\%). The specificity was estimated to be $91 \%$ (95\% CI: $75 \%$ 98\%). The positive predictive value of PET was $57 \%(95 \%$ CI: $18 \%-90 \%$ ), but there were only 7 patients classified as T4 by PET. The negative predictive value, on the other hand, was 63\% (95\% CI: 48\%-77\%).

PET scans were also analyzed to determine if the SUV correlated with $\mathrm{T}$ status. The mean SUV for the patients with T0 to T3 tumors was $6.7( \pm 2.9)$ and was also 6.7 ( \pm 3.9) for patients who had $\mathrm{T} 4$ disease. A receiver operating characteristic (ROC) curve based on SUV was constructed to determine the accuracy of FDG-PET in defining T4 disease (Figure 1). The AUC was 53\% ( $\pm 9 \%)$, indicating that FDG-PET SUV did not accurately predict $\mathrm{T}$ status.

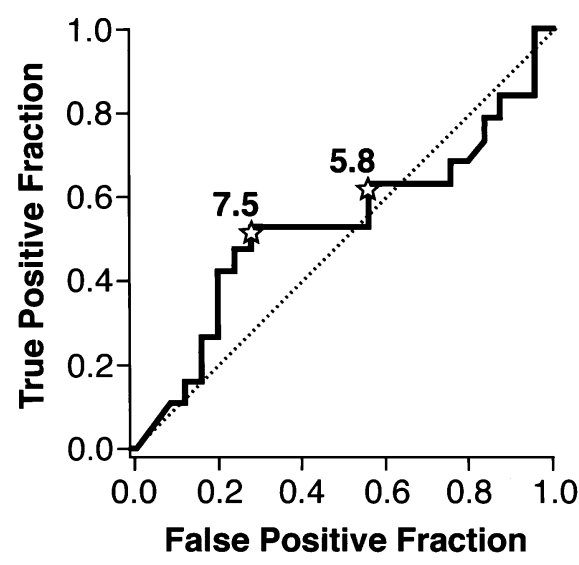

Figure 1. Receiver operator characteristic (ROC) curve of primary tumor standard uptake value (SUV) as a predictor of tumor T4 status. The area under the curve (AUC) of $53 \%$ indicates a poor accuracy of positron emission tomography (PET) in predicting tumor status.

TABLE 2. PET assessment of $\mathbf{N}$ status compared with the pathological $\mathbf{N}$ status

\begin{tabular}{lcccc}
\hline & \multicolumn{3}{c}{ N status by PET } \\
\cline { 3 - 5 } & & & & Total no. \\
& & N0 and N1 & N2 & patients \\
\hline Pathologic N status & N0 and N1 & 19 & 3 & 22 \\
Total no. patients & N2 & 8 & 1 & 9 \\
& & 27 & 4 & 31 \\
\hline
\end{tabular}

$N$, Nodal.

A correlation of PET findings to tumor $\mathrm{N}$ status was only possible in 31 patients, because the remaining patients technically could not have complete surgical evaluation by mediastinal lymph node dissection. As shown in Table 2, there was a total of 9 patients who had mediastinal nodal metastasis (N2 disease), but only 1 of these patients was correctly identified by PET. Two patients were considered to have hilar nodal metastasis (N1 disease), and the remaining patients were not considered to have any nodal metastasis by PET. Therefore, the sensitivity of PET in detecting nodal metastasis was $11 \%$ (95\% CI: $0 \%-48 \%$ ), and the specificity was $86 \%$ (95\% CI: $65 \%-97 \%$ ). The positive predictive value of PET was $25 \%$ (95\% CI: $1 \%-81 \%)$, and the negative predictive value was $70 \%$ (95\% CI: 50\%-86\%).

PET scans were also analyzed to determine if SUV of the primary tumor correlated with $\mathrm{N}$ status. The mean SUV for the 18 patients classified as $\mathrm{N} 0$ and $\mathrm{N} 1$ was $5.3 \pm 2.1$, and the mean SUV for the 9 patients who were classified as N2 positive was $8.6 \pm 3.4$. An ROC curve based on primary tumor SUV was constructed to determine the utility of FDG PET in defining N2 disease (Figure 2). The AUC was $78 \%$ 


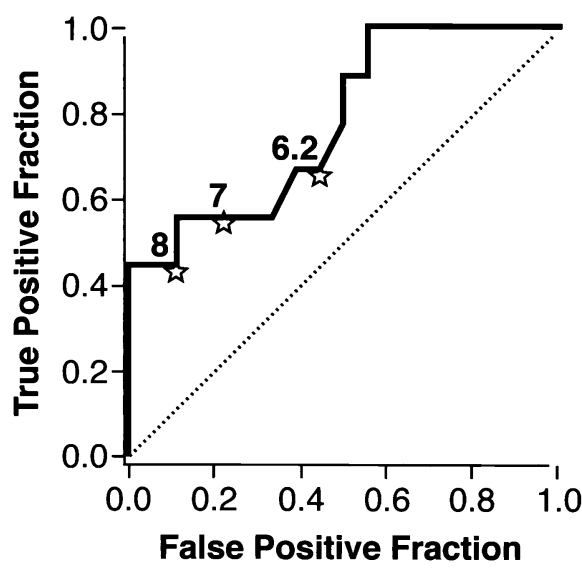

Figure 2. ROC curve of SUV of the primary tumor as a predictor of N2 status. The AUC of $78 \%$ seems potentially useful in predicting the presence of mediastinal nodal disease.

$\pm 10 \%$. The ROC curve is better for the $\mathrm{N}$ status than for the $\mathrm{T}$ status, and there seems to be an association between increased SUV and nodal disease. This indicates that patients who have a higher SUV in the primary tumor are more likely to have mediastinal nodal metastasis.

PET correctly identified 6 patients who had unresectable tumor because of extrathoracic disease. Open biopsy confirmed peritoneal metastasis in 1 patient, supraclavicular lymph node biopsy identified N3 disease in 2 patients, MRI confirmed pelvic bone metastasis in 1 patient, and PET identified N3 disease in 1 patient who developed a contralateral effusion while awaiting supraclavicular lymph node biopsy (and subsequent thoracentesis demonstrated metastatic mesothelioma). One patient with N3 disease, but no gross adenopathy on CT scan, underwent thoracotomy and proved to be unresectable on the basis of diffuse chest wall invasion.

PET failed to accurately detect disease in 3 patients. One patient had a false-negative scan that failed to detect a $1-\mathrm{cm}$ nodule of mesothelioma; the small size of the nodule on the CT scan was presumed to be the reason for the lack of detection by PET. Two patients had false-positive findings on PET scan (1 patient had a supraclavicular node that showed inflammation, which may have accounted for the false-positive results).

\section{Discussion}

Inaccurate clinical staging by current imaging modalities hinders the diagnosis and treatment of MPM. CT and MRI fail to identify unresectable disease in approximately $20 \%$ to $30 \%$ of patients. Our results confirm that most cases of MPM show increased FDG uptake on PET. We observed only 1 patient whose tumor did not have increased FDG uptake at initial diagnosis, and this patient was found to have an extremely early stage tumor. In addition, a falsenegative PET scan was obtained in a follow-up patient who developed a minimal amount of recurrent pleural tumor. Therefore, PET may fail to detect minimal tumor burden in MPM, and a negative PET scan does not absolutely rule out the presence of disease. In contrast with studies suggesting that PET can be used to distinguish between benign and malignant pleural disease, ${ }^{7}$ our results indicate that thoracoscopic biopsy may be necessary in such cases for definitive diagnosis.

The tumor histologic subtype does not seem to influence PET findings. Both epithelial and mixed histology tumors had similar SUVs. Although both of these tumors had lower SUVs than tumors of other histologies, there were too few tumors of sarcomatous subtype for analysis.

Given the lack of spatial localization provided by PET, it is not surprising that we did not reliably identify patients who were unresectable by virtue of T4 disease. The poor sensitivity of PET indicates that it should not be used to make decisions about the resectability of the primary tumor. It remains to be seen whether the newly available combined PET/CT scanners will provide better anatomic tumor definition.

We elected not to evaluate the influence of CT scan on staging. This study was performed specifically to correlate PET scan findings as a single modality with surgical pathologic findings. For staging to be considered, it would have required a retrospective review of different quality CT scanners from different institutions performed at different time periods other than the PET scan. Because the CT scans were performed in a non-uniform fashion, we would not have had the consistency that would be required to answer the specific question in a scientific manner. Combined PET/CT scanners are now available in many institutions and may provide better spatial localization and, therefore, improved staging of locoregional disease. This warrants future investigation.

The SUV was also not a good predictor of the T4 status. On the basis of the ROC curve, it seems that the SUV has no relationship at all to T status. The SUV probably reflects the rate of tumor growth and biology rather than a particular stage at the time of diagnosis.

It has been proposed that PET is useful in the determination of $\mathrm{N}$ status. ${ }^{6}$ Improved nodal staging would be valuable in MPM because of the known adverse impact of N2 disease on prognosis. ${ }^{4}$ However, our experience indicates that PET also does not reliably predict $\mathrm{N}$ status. This is not surprising given the difficulties in spatial resolution within the mediastinum. Pleural tumor involving or abutting the mediastinum will show increased FDG uptake and can easily be misinterpreted as nodal disease. There were a total of 9 patients with N2 disease, and PET did not identify 8 . Therefore, any management decision based on PET findings 
of N2 disease should be confirmed by surgical pathologic evaluation.

A high SUV in the primary tumor seems to correlate with the presence of mediastinal nodal metastasis. This may simply reflect the propensity of more metabolically active tumors to metastasize to mediastinal nodes. This is an interesting preliminary finding. Further investigation is needed to determine if there is a specific SUV level at which mediastinal nodal metastasis is likely to be present. Such information may be of potential use in selecting patients for surgical resection.

Our data indicate that FDG PET may be useful in identifying extrathoracic disease either preoperatively or in the setting of potential tumor recurrence. Although these findings warrant confirmation in a larger number of patients, it is valuable to identify even a small number of patients in whom surgical intervention may be inappropriate.

This experience builds on that reported in previous studies. The number of patients in our study is larger than in previously published studies, but it is still a somewhat heterogenous patient cohort. Additional studies in still larger numbers of patients are warranted. Future studies should investigate our observation that high primary tumor SUV correlates with N2 disease and could validate the lack of correlation between SUV and histologic subtype. Investigations of the accuracy of combined PET/CT scanning should also be undertaken to determine whether this imaging modality leads to better definition of $\mathrm{T}$ and $\mathrm{N}$ status.

\section{References}

1. Heelan RT, Rusch VW, Begg CB, Panicek DM, Caravelli JF, Eisen C. Staging of malignant pleural mesothelioma: comparison of CT and MR imaging. Am J Radiol. 1999;172:1039-47.

2. Patz EF Jr, Shaffer K, Piwnica-Worms DR, Jochelson M, Sarin M, Sugarbaker DJ, et al. Malignant pleural mesothelioma: value of CT and MR imaging in predicting resectability. Am J Roentgenol. 1992; 159:961-6.

3. Rusch VW, Venkatraman E. The importance of surgical staging in the treatment of malignant pleural mesothelioma. $J$ Thorac Cardiovasc Surg. 1996;111:815-26.

4. Sugarbaker DJ, Flores RM, Jaklitsch MT, Richards WG, Strauss GM, Corson JM, et al. Resection margins, extrapleural nodal status, and cell type determine postoperative long-term survival in trimodality therapy of malignant pleural mesothelioma: results of 183 patients. J Thorac Cardiovasc Surg. 1999;117:54-65.

5. Rusch VW, Rosenzweig K, Venkatraman E, Leon L, Raben A, Harrison L, et al. A phase II trial of surgical resection and adjuvant high dose hemithoracic radiation for malignant pleural mesothelioma. J Thorac Cardiovasc Surg. 2001;122:788-95.

6. Schneider DB, Clary-Macy C, Challa S, Sasse KC, Merrick SH, Hawkins R, et al. Positron emission tomography with f18-fluorodeoxyglucose in the staging and preoperative evaluation of malignant pleural mesothelioma. J Thorac Cardiovasc Surg. 2000;120:128-33.

7. Bénard F, Sterman D, Smith RJ, Kaiser LR, Albelda SM, Alavi A. Metabolic imaging of malignant pleural mesothelioma with fluorodeoxyglucose positron emission tomography. Chest. 1998;114:713-22.

8. Rusch VW. The International Mesothelioma Interest Group. A proposed new international TNM staging system for malignant pleural mesothelioma. Chest. 1995;108:1122-8.

9. American Joint Committee on Cancer. AJCC cancer staging handbook. 6th ed. New York, Berlin: Springer.
10. Union Internationale Contre le Cancer. TNM classification of malignant tumours. 6th ed. New York: Wiley-Liss, 2002.

\section{Discussion}

Dr David J. Sugarbaker (Boston, Mass). I very much appreciated the opportunity to read the article by Dr Flores and his group at Memorial regarding this interesting study of PET and mesothelioma. I think it is important to look for different modalities to understand preoperatively the extent of disease in mesothelioma, and I suggest that the findings in this particular study are not unexpected. The difficulty of defining the preoperative stage in mesothelioma is derived from the fact that the geographical distribution of the tumor, unlike other malignancies, is very diffuse, and all of the modalities, including MRI and CT scan (which preceded PET), have had difficulty in defining preoperatively the appropriate stage. Nevertheless, this study suggests that there are some limited useful applications of PET in mesothelioma, particularly in those malignancies that have a high metabolic rate taking up a lot of the markers; thus, there is the implication of a very high mitotic index and therefore a higher propensity to spread to the lymph nodes. Unfortunately, there is a very small group of patients who will demonstrate distant metastatic disease at the time of presentation. Nevertheless, in this small group of patients, it seems that PET has some usefulness.

I have 3 questions for Dr Flores and his group at Memorial, who obviously have extensive experience with mesothelioma. First, I believe 21 patients in your series were "open and close," unresectable. Given the limited cohort you had, this seems to be a relatively high unresectability rate. Would you address that and tell us if there was anything in the MRI, CT, or other aspects of the preoperative workup that may have indicated that these patients were indeed unresectable before their exploration? As a sideline to that question, was there a propensity for these patients to be mixed or sarcomatous types as opposed to epithelial types?

My second question concerns your statement that MRI is unreliable in determining resectability. Some years ago at Brigham, I, Dr Patz, and some of our colleagues in radiology wrote about the fact that although MRI is somewhat limited, it is the most useful preoperative staging radiologic modality when coupled with echocardiography. This is primarily because MRI allows one to see 2 important areas more easily and accurately than CT scanning: transdiaphragmatic extension of the disease and transmediastinal invasion into structures that are clearly going to render the patient unresectable. So, I ask you to comment on your use of MRI and echocardiography at Memorial in the workup of these patients.

Third, would you comment on the presence or absence of chest pain in your patients who are unresectable? What is your use of mediastinoscopy in the preoperative workup of these patients? We have found that even mild chest pain is often the best predictor of diffuse chest wall invasion by these tumors.

I thank Dr Flores and his group and congratulate them on an excellent study, which I think contributes to our knowledge of mesothelioma and to our understanding of the use of PET scanning in this disease.

Dr Flores. I will start by combining the first and third questions. In regard to the unresectability rate, many PET scans performed in patients were not obtained uniformly. Initially, more PET scans were performed in patients who had a higher tumor 